\title{
Perforations of Gastro Intestinal Tract in Neonates: A Retrospective Study of Aetiological Factors Excluding Necrotising Enterocolitis
}

\author{
Saravanan Natarajan,* Shankar Mohan, Sulthana Dhilras, Jameel Ahmed, Selvakumar Mariappan \\ Department of Pediatric Surgery, Govt. Medical College Hospital, Salem, Tamilnadu
}

This is an open-access article distributed under the terms of the Creative Commons Attribution License, which permits unrestricted use, distribution, and reproduction in any medium, provided the original work is properly cited.

\begin{abstract}
Background: Perforations of the Gastro Intestinal Tract (PGIT) in neonates, postnatally, apart from Necrotising Enterocolitis (NEC) as aetiological factor, though less common, are well known.

Materials and Methods: Neonates presenting with PGIT, excluding NEC, were analyzed in this retrospective study, over a period of three years in a tertiary care centre.

Results: Of 20 neonates presented with PGIT, during study tenure, 8 were due to non-NEC related causes. The site of perforation was stomach, ileum, cecum, colon, and rectum. The usual causes of PGIT in the neonates were spontaneous perforations and perforations secondary to mechanical obstruction. Patients underwent surgery with good outcome.

Conclusions: PGIT in neonates due to aetiological factors apart from NEC, have better outcome, than those with NEC. The other causes of PGIT are spontaneous perforation, intestinal atresia, Hirschsprung's disease, anorectal malformations etc.
\end{abstract}

Key words: Intestinal perforation; Neonate; Aetiology; Prognosis

\section{INTRODUCTION}

PGIT in neonates due to NEC is well known; other causes are usually spontaneous perforations or perforations secondary to mechanical obstruction.[1]

Spontaneous or focal intestinal perforations have no demonstrable cause, and the usual site of perforation is terminal ileum.[2] Mechanical obstruction may cause perforation at any part of the gastro intestinal tract. This study is performed to identify aetiological factors of PGIT in neonates apart from NEC.

\section{MATERIALS AND METHODS}

Over a period of three years from August 2015 to August 2018, neonates treated for PGIT in a medical college hospital were studied. Those that showed clinical and operative findings suggestive of NEC were excluded. Neonates born with meconium peritonitis were excluded.

There were total of 20 neonates treated for PGIT during the study period. Amongst them, 10 were due to NEC and 2 were antenatal perforations presenting as meconium peritonitis, and they were excluded from the study.

The remaining 8 neonates were taken up for this retrospective study. Age, sex, weight, maturity, time delay in presenting to the hospital, site of perforation, underlying pathology, aetiology, the management and complications, were studied in detail..

\section{RESULTS}

In this study, 5 neonates were males and 3 were females. Age of presentation varied from 2 days to 19 days. Three of them were premature. Prematurity varied from 32 weeks to 36 weeks. Common clinical findings were, abdominal distension, vomiting, and failure to pass meconium. Pneumoperitoneum was present in all the cases. All the neonates except two underwent laparotomy within 24 hours of presentation. Peritoneal drains were used only for initial management in 2 patients. The sites of perforations were found to be, Ileum in 4 cases, Caecum in 2 cases, Rectum in 1 case, and Stomach in 1 case. 
Table 1: Summary of the study population

\begin{tabular}{|l|l|l|l|l|l|l|l|}
\hline Sr. No. & Sex & Age (d) & Term & Wt (KG) & Perforation & Pathology & Management \\
\hline 1. & F & 2 & PT (36W) & 2.8 & Ileum & SP & Perforation closure \\
\hline 2. & M & 19 & FT & 3.5 & Ileum & SP & Resection \& anastomosis \\
\hline 3. & M & 7 & FT & 3.2 & Caecum & $\begin{array}{l}\text { Short } \\
\text { segment HD }\end{array}$ & $\begin{array}{l}\text { Perforation closure \& } \\
\text { ileostomy }\end{array}$ \\
\hline 4. & M & 3 & FT & 3.0 & Ileum & Ileal atresia & $\begin{array}{l}\text { Resection \& end to back } \\
\text { anastomosis }\end{array}$ \\
\hline 5. & M & 3 & PT (32W) & 2.6 & Rectum & ARM & $\begin{array}{l}\text { Perforation closure \& } \\
\text { transverse colostomy }\end{array}$ \\
\hline 6. & F & 15 & FT & 3.4 & Ileum & $\begin{array}{l}\text { Ovarian } \\
\text { cyst torsion }\end{array}$ & $\begin{array}{l}\text { Oophorectomy, resection } \\
\text { \& anastomosis }\end{array}$ \\
\hline 7. & M & 2 & PT (32W) & 2.6 & Stomach & SP & Repair \\
\hline 8. & F & 10 & FT & 3.2 & Caecum & TCA & $\begin{array}{l}\text { Resection of caecum \& } \\
\text { ostomies }\end{array}$ \\
\hline
\end{tabular}

TCA: Total colonic aganglionosis, SP: Spontaneous perforation

Four neonates had perforations in the ileum. In two of them there was no demonstrable cause, hence they were classified under spontaneous perforations. Both the neonates had presented to the hospital within 48 hours of onset of symptoms. Out of these two cases, one neonate with spontaneous ileal perforation, on laparotomy, was found to have single small perforation in the terminal ileum, which was managed by perforation closure. The other neonate had to undergo peritoneal drainage as initial procedure. This neonate subsequently underwent laparotomy the next day; resection of perforated part of ileum and ileo-ileal anastomosis was done. Both these neonates had uneventful recovery.

Of the other two cases with ileal perforation, one neonate had ileal atresia type III A which had perforation on account of delayed presentation (third day of life). This neonate had severe peritoneal contamination and after adequate peritoneal wash, an end to back anastomosis was done during laparotomy. But the neonate developed anastomotic leak post operatively, and underwent re-exploration and double barrel ileostomy formation. He is on follow-up, awaiting ileostomy closure. Another neonate, antenatal scan suggestive of intra-abdominal cyst, was admitted with peritonitis (delayed presentation, after 48 hours of onset of symptoms).

Laparotomy found torsion of right ovarian cyst, which got adherent to ileum producing volvulus, necrosis and perforation of ileum (Fig. 1). This neonate underwent Oophorectomy, resection and anastomosis of ileum. Except for postoperative wound infection, the neonate had good recovery.

Two cases of caecal perforations (Hirschsprung's disease (HD), as confirmed on histopathology. One neonate had a small caecal perforation, and transition zone was at the recto sigmoid region. This case underwent perforation closure and ileostomy. The other neonate had a large caecal perforation and gross intraperitoneal contamination, thus underwent resection of caecum and ostomy. The histopathology report of this neonate was in favour of Total Colonic Aganglionosis (TCA). This neonate also had post-operative wound infection, which was treated. Both of these neonates are on our follow-up for definitive procedures.

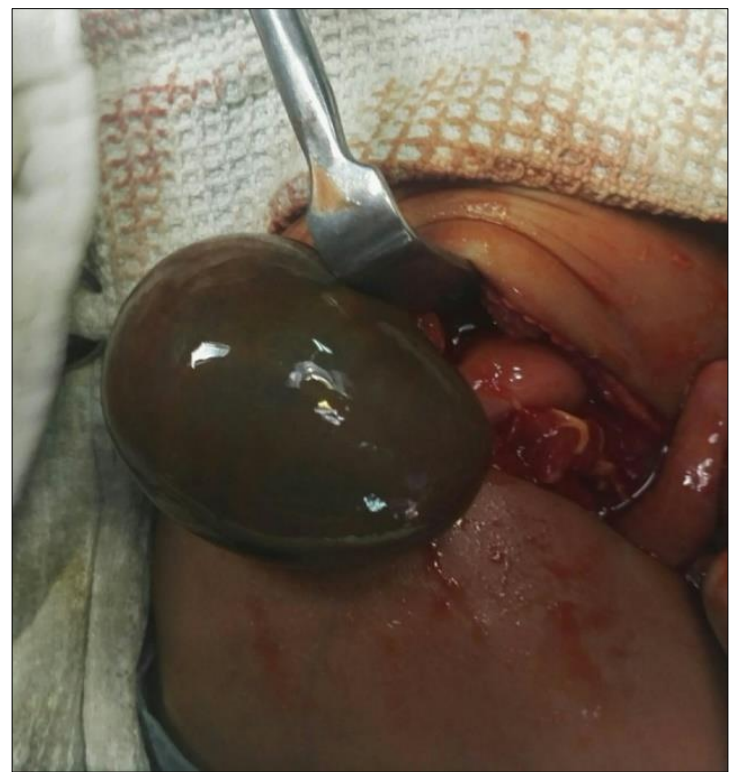

Figure 1: Twisted and necrosed ovarian cyst.

One neonate with rectal perforation was a preterm, twin delivery, referred on 3rd day of life, with missed low Anorectal Malformation (ARM. He underwent laparotomy, perforation closure and transverse colostomy. Post-operatively, this neonate also 
had wound infection, which was treated conservatively and improved. Subsequently he underwent posterior triangular anoplasty, and is awaiting colostomy closure.

The neonate with gastric perforation was also preterm with birth asphyxia; had undergone vigorous resuscitative measures with bag and mask, and was then subsequently given ventilator support. Peritoneal drainage was done and baby stabilised. Laparotomy was done the next day, which revealed gastric tear in the greater curvature, and that was repaired. This neonate died on the third postoperative day.

\section{DISCUSSION}

The first report of gastro-intestinal perforation in newborn was published by Siebold in 1825. PGIT in neonates most commonly occurs as a complication of NEC (42\%), and is associated with a high mortality rate $(62 \%) .[3,4]$ Apart from NEC, the common causes for neonatal PGIT are spontaneous perforations and mechanical obstruction. This was reflected in our study too; out of 20 cases of PGIT, 8 of them (40\%) belonged to this category. Compared to NEC, the mortality rate was only $12.5 \%$ in our study.

Gastric perforations in neonates account for approximately $7 \%$ of PGIT, and have poor prognosis and high mortality.[5,6] These perforations were historically thought to occur spontaneously, but it has been proven that distal obstruction is also a common cause for neonatal gastric perforations. Factors associated with neonatal gastric perforations include prematurity, asphyxia, congenital anomalies, stress at birth, vigorous respiratory measures, increased intra-gastric pressure, and anatomical abnormalities.[7] There was only one neonate in our series with gastric perforation, and that case also had multiple risk factors. The aetiology could either be spontaneous perforation or due to vigorous resuscitation. This neonate succumbed post operatively.

Spontaneous intestinal perforations are the second most common cause for PGIT in neonates.[3] Spontaneous or focal intestinal perforations are perforations with no demonstrable cause. Usually they affect very low birth weight preterm babies, but it has been noted also in term babies, and the usual site of perforation is terminal ileum.[2] Closed peritoneal drainage has been suggested as primary management followed by laparotomy, or as a definitive procedure.[8,9] Spontaneous intestinal perforation is a distinct clinical entity and has better outcome.[10] In our study, there were two neonates with spontaneous ileal perforation.
Mechanical obstruction is the other major cause of PGIT in neonates. According to the site of obstruction, the perforation can be at any part of the gastro intestinal tract. There were two cases of ileal perforations secondary to mechanical obstruction in our study. One was a case of ileal atresia with perforation. Such a presentation appears to be rare, based on lack of reports in the literature.[11] This neonate presented after 48 hours to the hospital, and postoperatively had anastomotic leak. We feel that late presentation and severe intraperitoneal contaminations were the reason for complications. The other neonate, developed ileal perforation secondary to adhesive obstruction, caused by torsion of ovarian cyst. This neonate also had postoperative wound infection attributed probably to the delay in presentation.

In our series, there were two caecal perforations. Caecal perforation in the absence of other obvious mechanical cause is due to HD. Most of the caecal perforations occur in TCA, but is also known to occur in short segment HD.[12] In our study, one was due to short segment $\mathrm{HD}$, and the other, due to TCA.

Rectal perforation in a neonate born with ARM is not unknown.[13] The incidence is about $2 \%$, and it increases to $9.5 \%$ when diagnosis is delayed.[14] In our series also, the only neonate which had rectal perforation, the diagnosis was delayed, and presented on the third day of life with perforative peritonitis.

\section{CONCLUSION}

Spontaneous perforations and perforations secondary to mechanical obstruction are significant aetiological factors in the development of perforations of gastrointestinal tract in neonates. Their outcome is better, compared to perforations secondary to NEC.

Consent: Authors declared that they have taken informed written consent, for publication clinical photographs/material, from the legal guardian of the patient with an under-standing that every effort will be made to conceal the identity of the patient however it cannot be guaranteed.

Author Contributions: All the authors contributed fully in concept, literature review, and drafting of the manuscript and approved the final version of this manuscript.

\section{REFERENCES}

1. Hyginus EO, Jideoffor $U$, Victor $M$, et al.Gastrointestinal perforation in neonates: aetiology and risk factors. J Neonatal Surg .2013; 2:30.

2. Korakaki E, Manoura A, Hatzidaki E, Arbiros J, Vlahakis J, Valari V, et al. Spontaneous intestinal 
perforation in a full-term infant: association with infection. Minerva Pediatr. 2003; 55:289-92.

3. Grosfeld JL, Molinari F, Chaet M, Engum SA, West KW, Rescorla FJ. Gastrointestinal perforation and peritonitis in infants and children. An experience with 179 cases over 10 years. Surgery 1996; 120:650-5.

4. Zamir O, Shapira SC, Udassin R, Peleg O, Arad I, Nissan S. Gastrointestinal perforations in the neonatal period. Am J Perinatol. 1988; 5:131-3.

5. St-Vil D, LeBouthillier G, Luks FI, Bensoussan AL, Blanchard H, Youssef S. Neonatal gastrointestinal perforations. J Pediatr Surg. 1992;b27:1340-2.

6. Tan CE, Kiely EM, Agrawal M, Brereton RJ, Spitz L. Neonatal gastrointestinal perforation. J Pediatr Surg. 1989; 24:888-92.

7. Byun J, Kim HY, Noh SY ,Kim SH, Jung SE, Lee SC, et al. .Neonatal gastric perforation: A single center experience. World J Gastrointest Surg. 2014; 6:1515.

8. Cass DL, Brandt ML, Patel DL, Nuchtern JG, Minifee PK, Wesson DE. Peritoneal drainage as definitive treatment for neonates with isolated intestinal perforation. J Pediatr Surg. 2000; 35:1531-6.

9. Demestre X, Ginovart G, Figueras-Aloy J, Porta R, Krauel X, Garcia-Alix A, et al. Peritoneal drainage as primary management in necrotizing enterocolitis: A prospective study. J Pediatr Surg. 2002; 37:963-7.

10. Tiwari C, Sandlas G, Jayaswal S, Shah H. Spontaneous intestinal perforation in neonates. J Neonatal Surg. 2015; 4:14.

11. McAdams RM, Ledbetter DJ. Ileal atresia and multiple jejunal perforations in a premature neonate with gestational alloimmune liver disease. J Pediatr Surg Case Rep. 2017;19:48-51.

12. Singh S, Rawat J, Wakhlu A, Kureel SN, Pandey A. Six-year retrospective analysis of colonic perforation in neonates and infants: Single centre experience. Afr J Paediatr Surg. 2012; 9:102-5.

13. Sarin YK. Intra-peritoneal rectal perforation in a neonate leading to acquired rectal atresia. J Neonatal Surg. 2013; 2:22.

14. Turowski C, Dingemann J, Gillick J. Delayed diagnosis of imperforate anus: an unacceptable morbidity. Pediatr Surg Int. 2010; 26:1083-6.. 